$10-11-2006$

\title{
Quantifying Fluid Mixing with The Shannon Entropy
}

\author{
Marco Camesasca \\ Case Western Reserve University \\ Miron Kaufman \\ Cleveland State University, m.kaufman@csuohio.edu \\ Ica Manas Zloczower \\ Case Western Reserve University, ica.manas-zloczower@case.edu
}

Follow this and additional works at: https://engagedscholarship.csuohio.edu/sciphysics_facpub

Part of the Physics Commons

How does access to this work benefit you? Let us know!

Publisher's Statement

This is the accepted version of the following article: Marco Camesasca, Miron Kaufman and Ica Manas-Zloczower, "Quantifying Fluid Mixing with the Shannon Entropy," Macromolecular Theory and Simulations 15 (8), 595-607 (2006)., which has been published in final form at http://onlinelibrary.wiley.com/doi/10.1002/mats.200600037/abstract

\section{Repository Citation}

Camesasca, Marco; Kaufman, Miron; and Zloczower, Ica Manas, "Quantifying Fluid Mixing with The Shannon Entropy" (2006). Physics Faculty Publications. 238.

https://engagedscholarship.csuohio.edu/sciphysics_facpub/238

This Article is brought to you for free and open access by the Physics Department at EngagedScholarship@CSU. It has been accepted for inclusion in Physics Faculty Publications by an authorized administrator of EngagedScholarship@CSU. For more information, please contact library.es@csuohio.edu. 


\title{
Quantifying Fluid Mixing with the Shannon Entropy
}

\author{
Marco Camesasca, Miron Kaufman, Ica Manas-Zloczower
}

\section{Introduction}

Quantifying mixing is important both at the macroscopic scale for the optimization of industrial processes, such as mixing colorants in a polymer matrix or mixing of gases in an engine, and at the microscopic level when dealing with microfluidics such as microreactors and micromixers.

Fundamental concepts and ideas from mathematics and physics can be used to develop quantitative measures (for a recent and extended review see Wiggins and Ottino, 2004). ${ }^{[1]}$ The mechanisms for mixing are diverse. Diffusion provides mixing on a molecular scale, while at larger scales macroscopic flows are responsible for mixing: Aref has studied the use of chaotic advection to enhance mixing in laminar flows. ${ }^{[2]}$ Fluid mixing is also particularly important in microscience and nanoscience. ${ }^{[3-6]}$

Various techniques have been used to quantify mixing. Danckwerts ${ }^{[7]}$ has introduced the concepts of scale and inteneffectiveness is best achieved through modeling and simulations for equipment design and processing-parameter optimization.

\section{Mixing and Entropy}

In laboratory experiments, to study the mixing properties of a system a tracer is placed in the flow and passively advected; numerically this corresponds to putting a blob of particles in the flow field obtained by numerical solution of the equations governing the system. These particles, sometimes called passive scalars, are idealized: massless and small enough not to perturb the flow but large enough not to engage in Brownian motion (i.e. no diffusion). This procedure undoubtedly offers visual insights into the system and it can be implemented so that two or more tracers completely fill the system space. In this way it is possible to image the behavior of two or more fluids in time, granted that the fluids have the same physical and rheological properties. A point of terminology in the rest of the paper is sity of segregation that are widely used for mixing characterization. ${ }^{[8]}$ Chaotic mixing is inferred from Poincaré maps, ${ }^{[9]}$ periodic point analysis, ${ }^{[10]}$ or Lyapunov exponents. ${ }^{[9,11]}$ More-direct methods used are fluorescent spectroscopy ${ }^{[3,12]}$ or the calculation of the striation thickness or stretching distributions. ${ }^{[9,13,14]}$ Distributive mixing behavior of a given flow has been described in terms of standard deviation and maximum sample error. ${ }^{[15]}$ In spite of the large amount of work on this topic, there is no consensus on a single measure of mixing that can be used for different processes nor is there a single mixing index that can be implemented easily for different scenarios varying from numerical simulations to laboratory experiments to industrial processes. ${ }^{[16-19]}$ In this paper we present an approach for measuring the degree of mixing of several fluids that is independent of the physical process responsible for mixing and that relies on the information entropy. This approach is particularly suited for polymerprocessing operations where process control and enhanced that we use the word species rather than tracer because the latter is only associated with a color property whereas the former refers more generally to the possibility of distinguishing different particles without being limited by color.

Perfect mixing is characterized by the fact that in any spatial region the local concentration of any one component is the same as the concentration of that component in the whole system. ${ }^{[9]}$ Our entropic measure achieves its maximum for this perfect mixing situation and it is also able to give a quantitative characterization for mixing levels that are less than perfect. Whereas in chaotic systems perfect mixing is achievable, nonchaotic systems can also attain some degree of mixing at a given scale of observation. Quantifying mixing, perfect or not, as a function of the scale of observation, has important applications and constitutes the goal of this paper.

Our motivation for using Shannon informational entropy to measure mixing is rooted in its rigorous mathematical background. The Shannon entropy ${ }^{[20]}$ is uniquely determined from the Khinchin axioms, ${ }^{[21]}$ a set of properties 
that a measure of mixing should possess: (i) it depends on the probability distribution $p$ only; (ii) the lowest entropy $(S=0)$ corresponds to one of the $p$ s being 1 and the rest being zero (i.e., total information, perfect order, complete segregation); (iii) the largest value for the entropy is achieved when all $p$ s are equal to each other (i.e., the absence of any information, uniform distribution of the species everywhere in the system, perfect mixing); and (iv) $S$ is additive over partitions of the outcomes (i.e. the sum of entropies is still an entropy). Furthermore, the Shannon information entropy has been used extensively in many different scientific areas as a measure of disorder or lack of information. ${ }^{[22-26]}$ We have employed entropic measures for a variety of practical applications in mixing of polymer processing. ${ }^{[27-31]}$

The system is divided into 'bins' and for each bin $j, j=1$, 2. .M, we evaluate the number $n_{j, c}$ of particles of species $c$, $c=1,2 \ldots C$. The joint probability that a particle of species $c$

a)

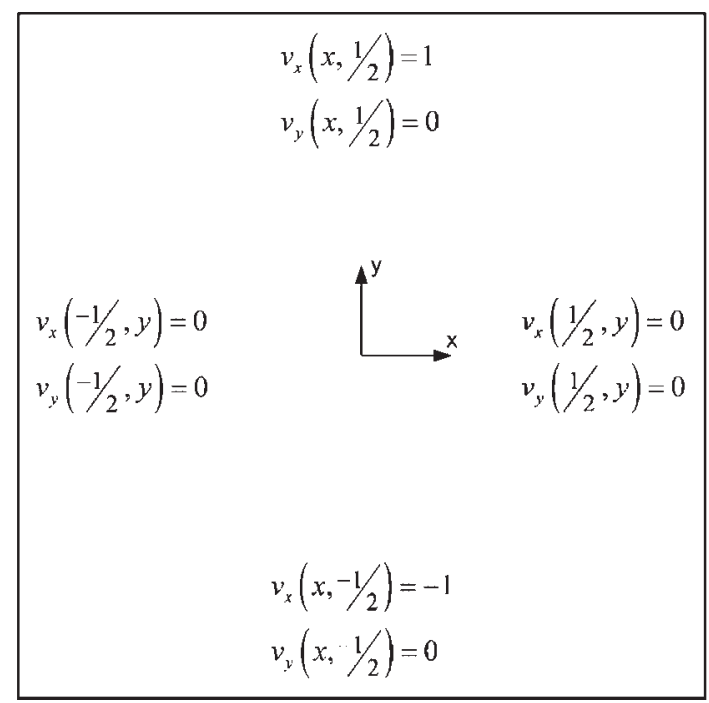

c)

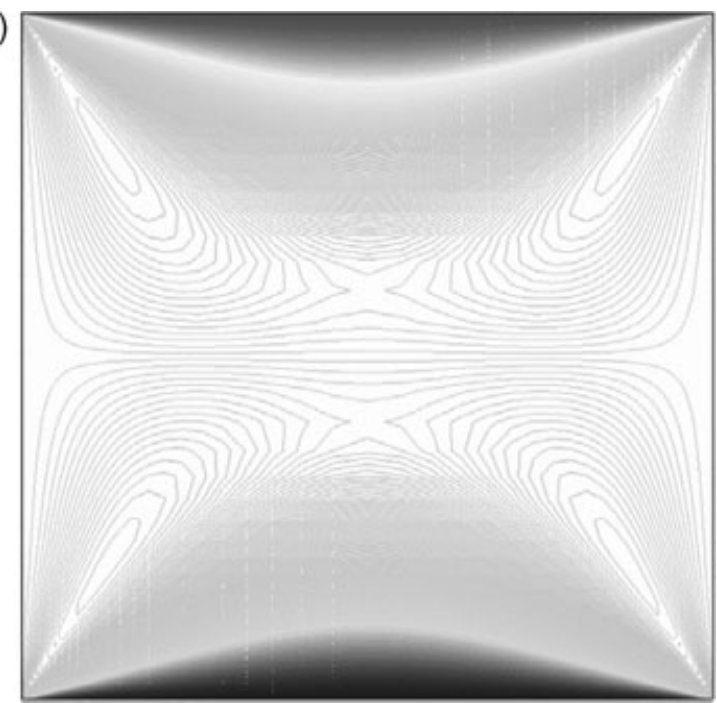

is in bin $j$ can be calculated by dividing $n_{j, c}$ by the overall system population. When the populations $n_{c}$ are not equal with each other but proportional to $P_{c}, c=1,2 \ldots C$, we calculate a joint probability to find a group/complex of $P_{c}$ particles of species $c$ in bin $j$ :

$$
p_{j, c}=\frac{\frac{n_{j, c}}{P_{c}}}{\sum_{i=1}^{M} \sum_{c=1}^{C} \frac{n_{i, c}}{P_{c}}} .
$$

Using the joint probabilities of Equation (1) we then calculate the entropy:

$$
S=-\sum_{j=1}^{M} \sum_{c=1}^{C} p_{j, c} \ln p_{j, c}
$$

b)

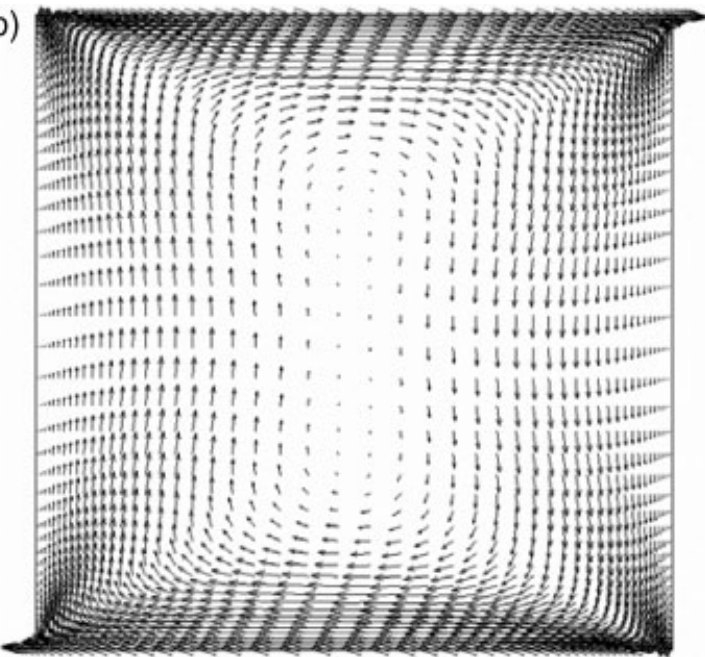

d)

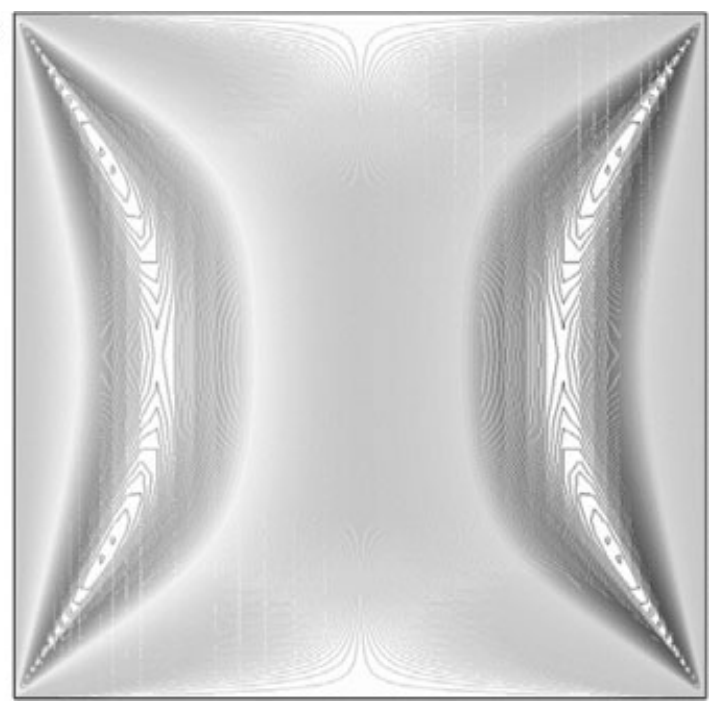

Figure 1. (a) Schematics of the cavity with boundary conditions. The upper and lower walls move along the $x$ coordinate with velocity $V \quad 1$ in opposite directions. (b), (c) and (d) Velocity field for the system and contours of $v_{x}(x, y)$ and $v_{y}(x, y)$. 
According to the third Khinchin axiom, the entropy achieves its maximum when all $p_{j, c}$ are equal to each other. In this case Equation (1) implies:

$$
\frac{n_{j, 1}}{P_{1}}=\frac{n_{j, 2}}{P_{2}}=\ldots=\frac{n_{j, C}}{P_{C}}=\frac{\sum_{c=1}^{C} n_{j, c}}{\sum_{c=1}^{C} P_{c}} j=1,2, \ldots, M .
$$

The significance of Equation (3) is that the entropy is maximized when at each location $j$ the fraction of each component is the same as in the whole system. This is the main characteristic of perfect mixing and this is the motivation to use the entropy to quantitatively characterize mixing.

In view of the fourth Khinchin axiom, the total entropy is additive. Thus, we can derive, from Equation (2), Equation (4) below which expresses the total entropy as the sum of two other entropies: the conditional entropy

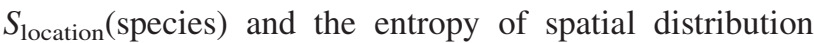
$\mathrm{S}$ (location). A detailed derivation is presented in the Appendix.

$$
S=S_{\text {location }}(\text { species })+S(\text { location }) \text {. }
$$

a)

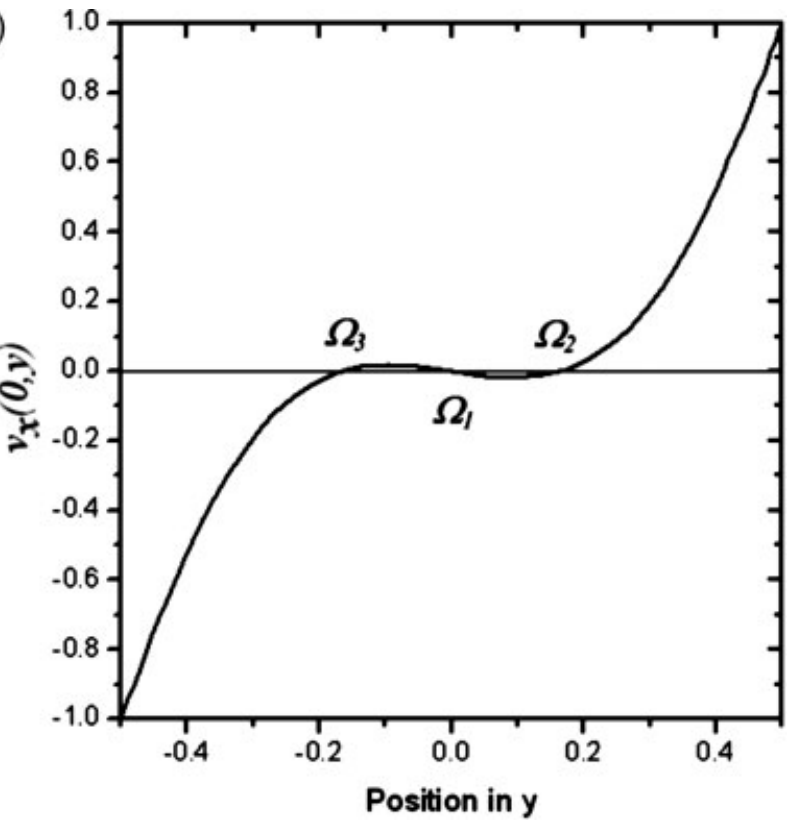

b)
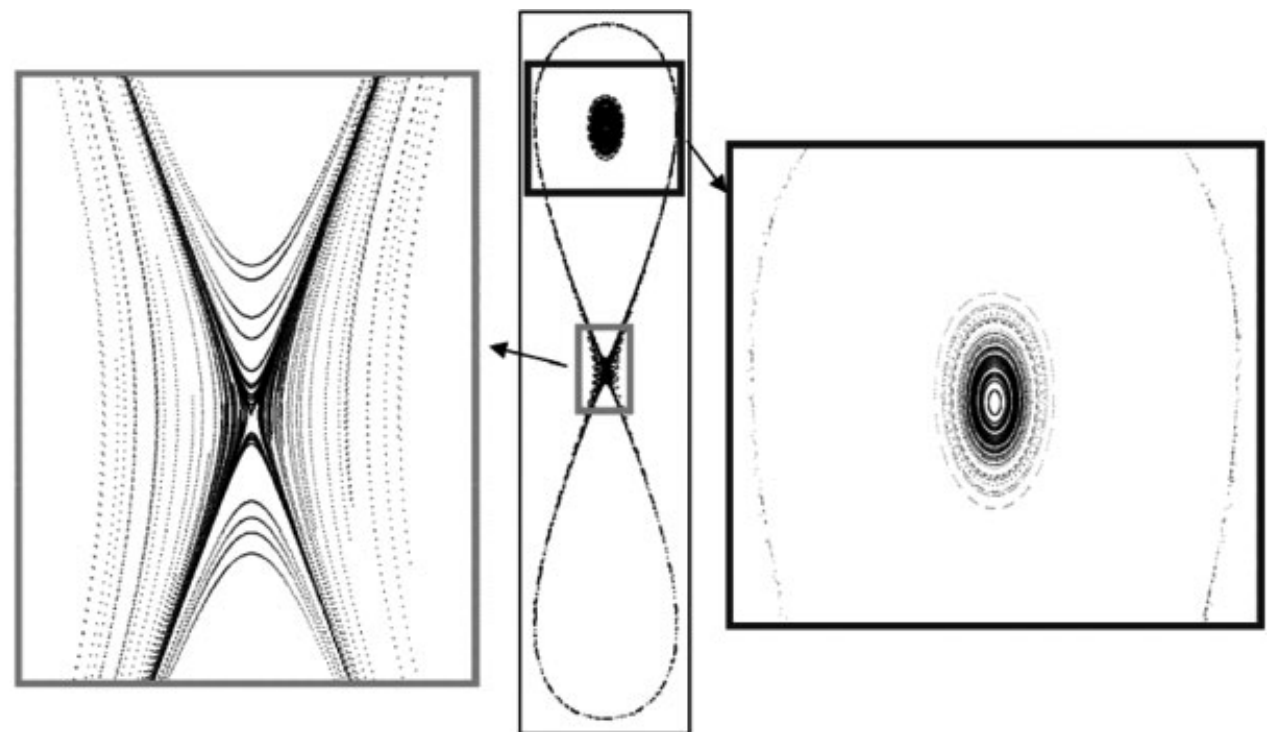

Figure 2. (a) $v_{x}(0, y)$ versus position in $y$. The three stagnation points $\Omega_{1}, \Omega_{2}, \Omega_{3}$ correspond to the intersections with the horizontal line. (b) Section of the system revealing the presence of the stagnation points $\Omega_{1}$ and $\Omega_{2}$. 
a)

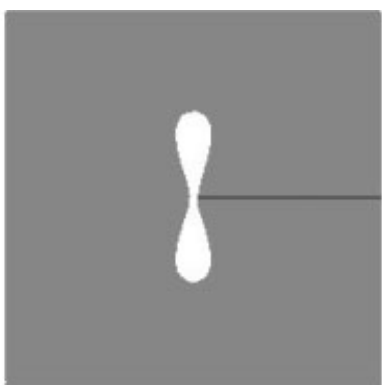

b)

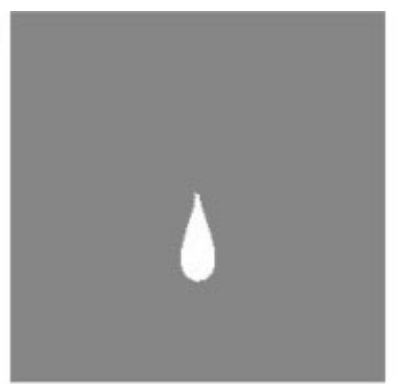

c)

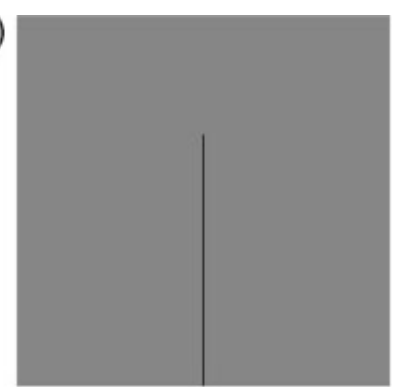

Figure 3. (a) Streamlines of 2000 particles initialized on the dark line intersecting one wall but not any of the stagnation points. (b) Streamlines of 2000 particles initialized on the dark line intersecting one wall and two stagnations points. (c) Streamlines of 2000 particles initialized on the dark line intersecting one wall and the three stagnations points.
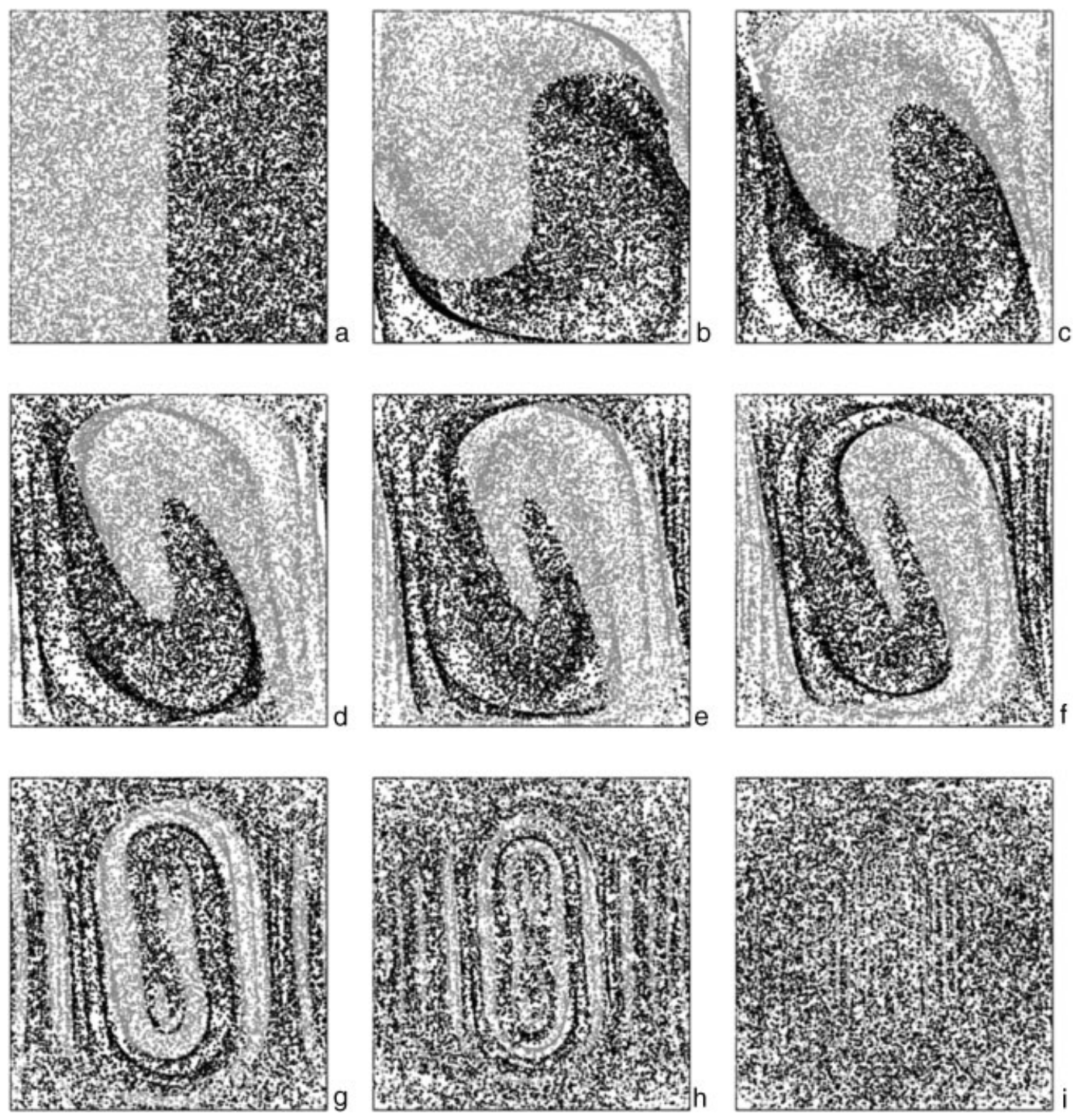

Figure 4. System 1: dynamics of mixing of two Newtonian, completely miscible fluids, represented by black and gray dots, with same overall concentration (equal number of particles for the two species is used in the simulation). (a) $t \quad 0$; (b) $t \quad 1$; (c) $t \quad 2$; (d) $t \quad 3$; (e) $t \quad 4$; (f) $t \quad 5$; (g) $t \quad 15$; (h) $t \quad 30$; (i) $t \quad 100$ in units of $a / V$. 


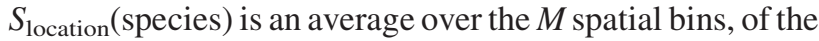
entropy of mixing the $C$ species conditional on bin location:

$$
S_{\text {location }}(\text { species })=\sum_{j=1}^{M}\left[p_{j} S_{j}(\text { species })\right],
$$

and

$$
S_{j}(\text { species })=-\sum_{c=1}^{C}\left[p_{c / j} \ln p_{c / j}\right] .
$$

In Equation (5) and (6) $p_{j}$ is the probability that a complex/group of particles, irrespective of species composition, is in bin $j$ and $p_{c / j}$ is the conditional probability to find a complex of $P_{c}$ particles of species $c$ conditioned on being in bin $j$. $S$ (location) is the entropy associated with the spatial distribution of particles irrespective of their species:

$$
S(\text { location })=-\sum_{j=1}^{M} p_{j} \ln p_{j} .
$$

The probabilities $p_{c / j}$ and $p_{j}$ are estimated using:

$$
p_{c / j}=\frac{\frac{n_{j, c}}{P_{c}}}{\sum_{c=1}^{C} \frac{n_{j, c}}{P_{c}}},
$$
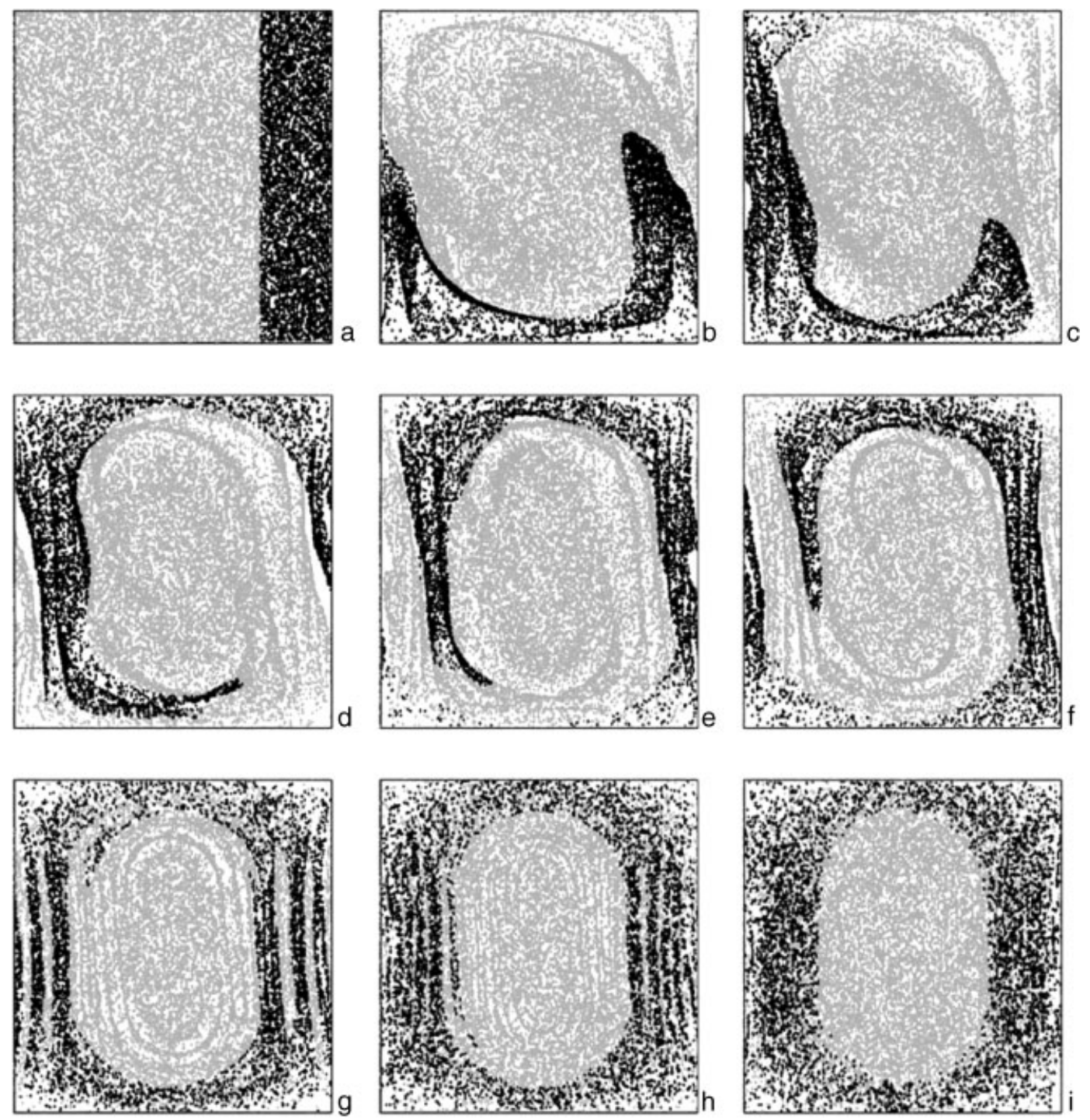

Figure 5. System 2: dynamics of mixing of two Newtonian, completely miscible fluids, represented by black and gray dots, with different overall concentration. This case differs from the one in Figure 4 in that the overall ratio of the two fluids is $3: 1$. (a) $t=0$; (b) $t=1$; (c) $t=2$; (d) $t=3$; (e) $t=4$; (f) $t=5$; (g) $t=15$; (h) $t=30$; (i) $\mathrm{t}=$ 100 in units of $a / V$. 
$S$ (location) quantifies the spatial homogeneity of the particles irrespective of their species. $S_{\text {location }}$ (species), on the other hand, quantifies the quality of mixing of the $C$ species conditional on locations. In the example used in the next section we look at an incompressible fluid carrying $C=2$ species of particles that are initially well distributed in space. Hence $S$ (location), normalized by $\ln M$ [which is, by construction, the maximum value that $S$ (location) can reach] is a time constant and the interesting measure that we concentrate on is $S_{\text {location }}$ (species), normalized by $\ln C$.

In practical situations the quality of mixing depends on the scale of observation: in polymer processing, for example, mixing of two polymers can be considered satisfactory when the striation thickness is uniformly of the order of a specific size $\lambda^{*}$ desired for the use of the final product (typically of the order of microns). However if one studies the mixing below that value, most likely the two polymers will still be segregated. More generally, when a real system is declared mixed it may be the case that it is as such only up to a certain scale $\lambda \geq \lambda^{*}$ and if the mathematical condition is checked for subregions smaller than that scale, then the system will not be mixed. For this reason it is important to have a measure of mixing that can be continuous from 0 (not mixing) to 1 (perfect mixing) so that different systems, at different times, may be compared.

We control the scale of observation by the number of equal-sized bins $M$ : as $M$ increases, the linear size of each $\operatorname{bin} \lambda$ decreases. If the segregation of the species occurs at a
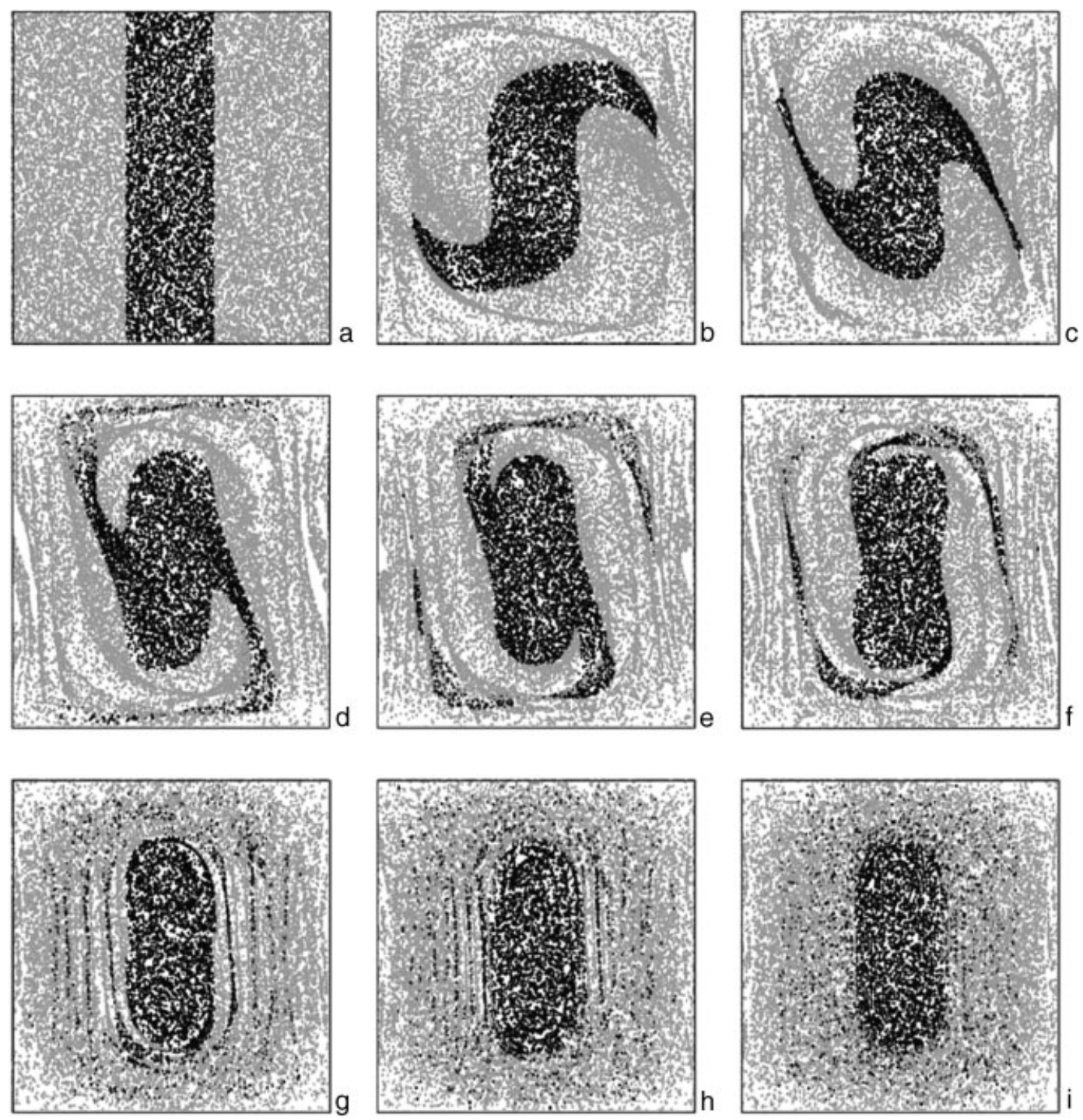

Figure 6. System 3: dynamics of mixing of two Newtonian, completely miscible fluids, represented by black and gray dots, with different overall concentration. This system differs from the one in Figure 5 only in the initial distribution of the two species. (a) $t=0$; (b) $t=1$; (c) $t=2$; (d) $t=3$; (e) $t=4$; (f) $t=5$; (g) $t=15$; (h) $t=30$; (i) $t=100$ in units of $a / V$. 
length scale $\lambda^{*}$, then as the scale of observation $\lambda$ is decreased a poorer mixing will be observed. In general it is expected to observe good mixing for $\lambda>\lambda^{*}$ and poor mixing when $\lambda<\lambda^{*}$.

\section{Case Study: Flow in a Cavity}

\section{Description and Numerical Simulations}

The system is a two-dimensional square cavity with two parallel walls moving in opposite directions and the other two walls stationary, as shown in Figure 1. The cavity has length $a$. Mixing in this system can be achieved by simple stirring even in the absence of chaotic advection, ${ }^{[9]}$ as this is the case since the flow is steady. Although time-dependent systems may present chaotic behavior we chose this configuration because it is simple, it mimics the action of stirring and it is akin to one common in polymer processing when a polymer is processed using the single-screw extruder equipment. The unwound channel model of the extruder corresponds to a rectangular duct with a moving top wall. ${ }^{[32]}$ It is also interesting per se because of the possibility of achieving different final configurations depending only on the initial positional conditions: if the system is chaotic, no matter what the initial conditions are they all will be the same after a long enough time with no different final results.

Numerical simulation of the system was performed using FIDAP, a fluid-dynamics software (CFD) based on the finite element method (FEM). Since in this work we are not concerned with the mechanisms of mixing, no other effects,
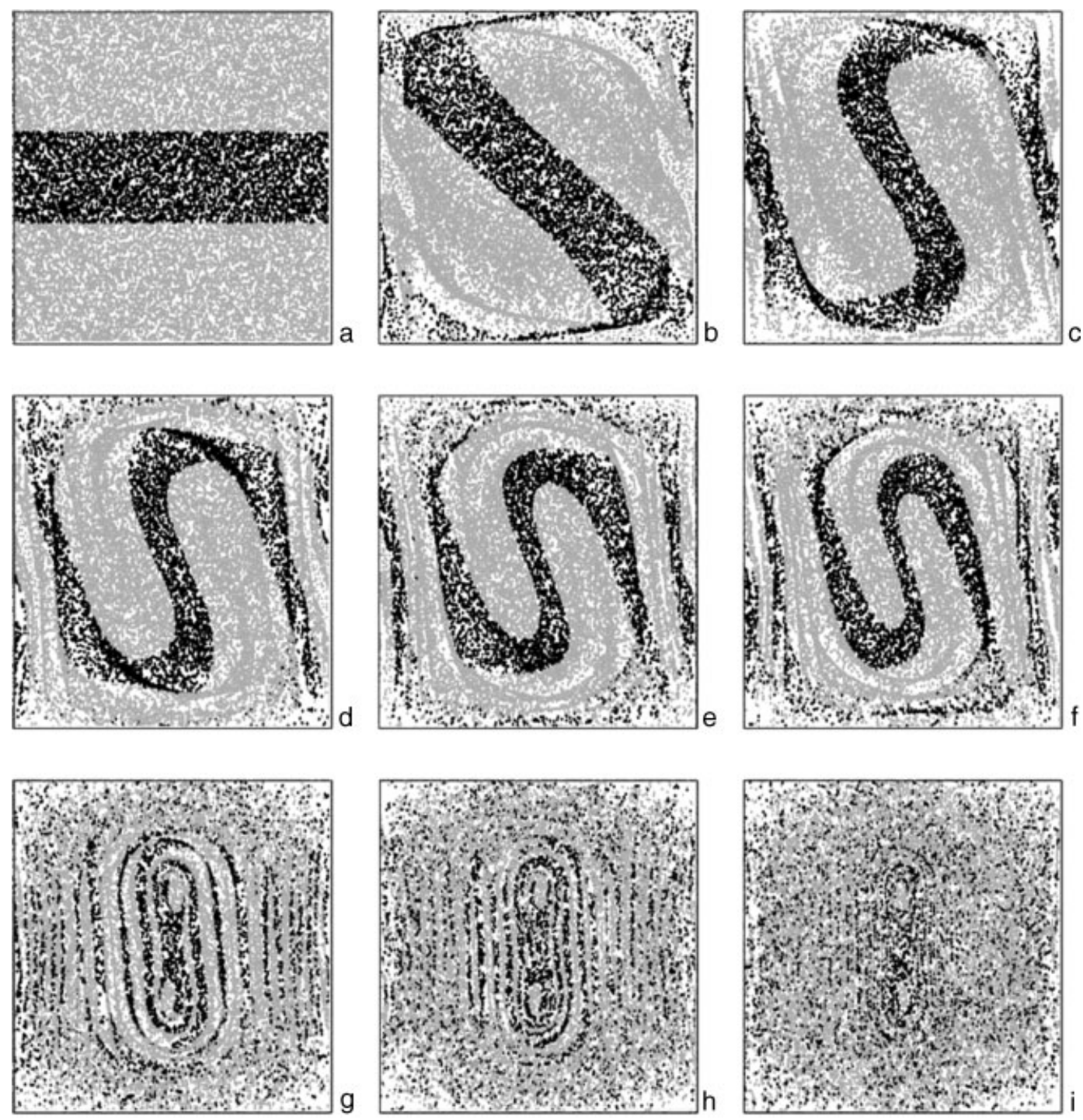

Figure 7. System 4: dynamics of mixing of two Newtonian, completely miscible fluids, represented by black and gray dots, with different overall concentration. Again, this system differs from those in Figure 5 and 6 only in the initial distribution of the two species. (a) $t=0$; (b) $t=1$; (c) $t=2$; (d) $t=3$; (e) $t=4$; (f) $\mathrm{t}=5$; (g) $t=15$; (h) $t \quad 30$; (i) $t \quad 100$ in units of $a / V$. 
such as diffusion, temperature gradients, or chemical interactions, have been considered in the simulation. In our model, mixing is solely produced by the flow field so that the problem is fully described by the equations of conservation of mass (continuity equation) and conservation of momentum (Navier-Stokes equation). There is no turbulence since we chose to simulate a system with Reynolds number, $R e=1$, well below the turbulence threshold. As already mentioned, the velocity is not a function of time, therefore no chaotic advection is induced and the striation thickness will possibly decrease only by the stirring action of the laminar flow.

The top wall moves to the right and the bottom wall moves to the left with the same speed $V$. By setting $V=1$ we express all velocities in units of $V$ and the time in units of $a / V$. he boundary conditions are:

top wall $\left\{\begin{array}{l}v_{x}(x, 0.5)=1 \\ v_{y}(x, 0.5)=0\end{array}\right.$ bottom wall $\left\{\begin{array}{l}v_{x}(x,-0.5)=-1 \\ v_{y}(x,-0.5)=0\end{array}\right.$

left wall $\left\{\begin{array}{l}v_{x}(-0.5, y)=0 \\ v_{y}(-0.5, y)=0\end{array} \quad\right.$ right wall $\left\{\begin{array}{l}v_{x}(0.5, y)=0 \\ v_{y}(0.5, y)=0\end{array}\right.$

In Figure 1 the velocity field is presented. The fluids rotate clockwise, symmetrically with respect to the lines of $x=0$ and $y=0$. Three stagnation points can be identified at positions $\Omega_{1}=(0,0)$ and $\Omega_{2,3}=(0, \pm 0.161)$ in units of $a$ (Figure 2).

Because of the nonchaotic and relatively simple configuration of the system, particle trajectories are calculated numerically since there is no analytical solution for the velocity field. The particle-tracking method used is the fourth-order Runge-Kutta with fixed time-step $\Delta t=10^{6} \times$ $a /$ Von the velocity field calculated using a segregated solver with pressure projection solution method for a structured mesh of quadrilateral elements comprised of 2601 nodes $(51 \times 51)$; to adapt the mesh refinement to the solution, the nodes are densified at the edges. We calculate the velocity values between nodal points by interpolation via a bicubic method.

To verify the presence of stagnation points we constructed sections of the system as illustrated in Figure 2(b). Notice the presence of one hyperbolic point at $(0,0)$ and 2 elliptic points at $(0, \pm 0.161)$. The positions of the stagnation points $\Omega_{2,3}$ are affected by the geometry of the cavity, namely the aspect ratio $h / w$, with $h$ the cavity height ( $y$ coordinate) and $w$ the cavity width ( $x$ coordinate). If the aspect ratio $h / w$ decreases, the distance between $\Omega_{2,3}$ and $\Omega_{1}$ decreases and eventually all the points collapse into $(0,0)$; while as $h / w$ increases the three points will move further and further apart. ${ }^{[32,33]}$

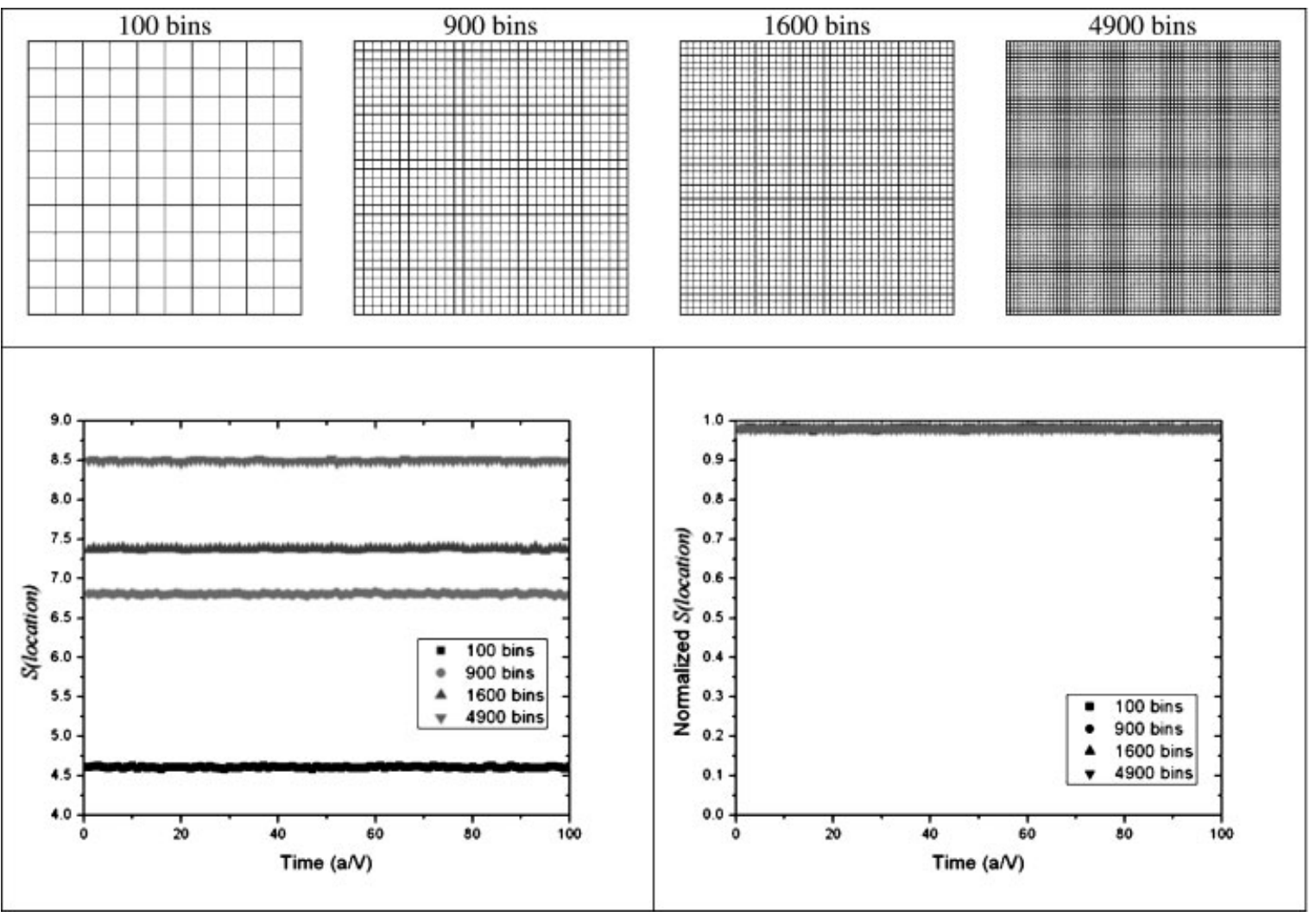

Figure 8. (top) Relative dimensions of the bins compared to the size of the cavity; (bottom left) $S$ (location) versus time for system 1 at different number of bins: 100, 900, 1600, 4900; (bottom right) normalized $S$ (location) for the four systems; all normalized $S$ (location) have values very close to 1 and are indistinguishable. 


\section{System Behavior and Mixing Imaging}

Based on the above observations, it follows that to guarantee a complete 'filling' of the system by the particle streamlines, tantamount to perfect mixing/spreading of these particles in the system, one would have to seed particles on a vertical line connecting the two points $\Omega_{2}$ and $\Omega_{3}$ with a wall. If such a line is used as a guideline for placing the interface between two separated fluids, the initial placement will permit total mixing of the fluids since as time progresses they will be equally spread in the entire space. In this case, in the limit of infinite time, the striation thickness will tend to zero. In Figure 3 we illustrate this point by showing the streamlines of 2000 particles initially placed on different straight lines. If the particles are initially placed on lines which do not contain all three stagnation points and intersect either the upper or lower wall, the streamlines generated will not cover the entire cavity surface [Figure 3(a) and 3(b)]. When particles are placed

a)

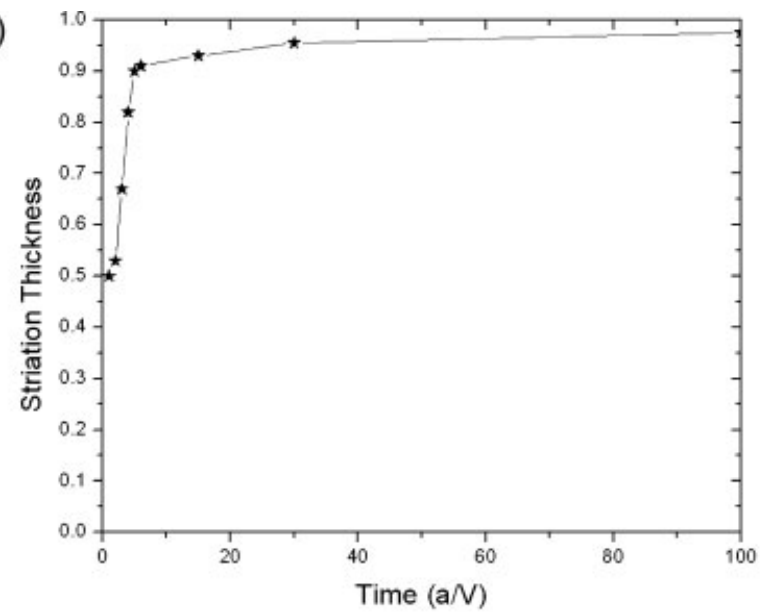

b)

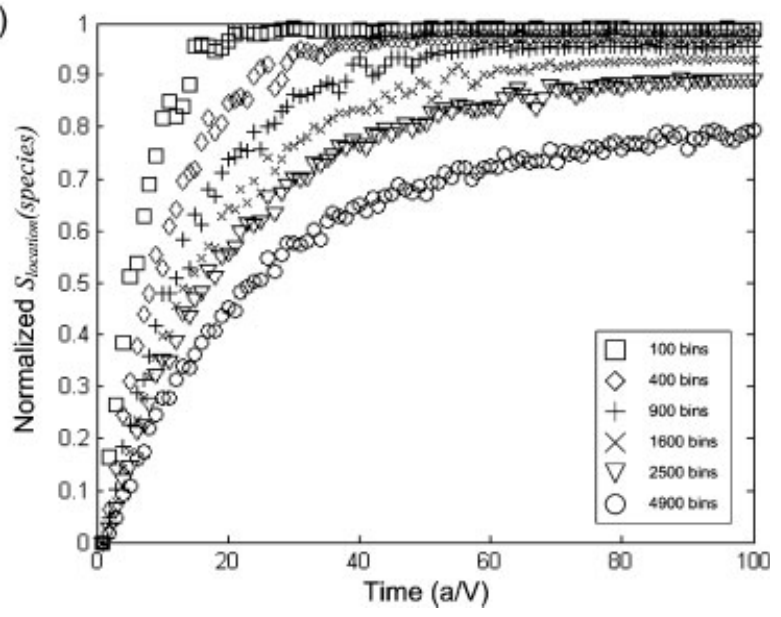

Figure 9. (a) (1 striation thickness) versus time for system 1. (b) Normalized $S_{\text {location }}$ (species) versus time for system 1 calculated at six different numbers of bins $(100,400,900,1600$, 2500, 4900): the behavior of the entropy is strongly dependent on the number of bins; the maximum value is reached only at a large scale of observation (i.e. 100 bins). initially on a line that touches one wall and intersects all the stagnation points, the streamlines fill the entire space as shown in Figure 3(c).

In order to illustrate the mixing dynamics in our system we use 20000 particles of the two species, randomly placed in the entire area to completely fill it. In our analysis we employ the conventional forward-particle-tracking method, although it should be mentioned that an alternative procedure based on the backward tracking may provide higher quality images. ${ }^{[8,34,35]}$ Four case scenarios are considered. In the first one, 10000 particles per species are placed randomly and evenly in the square cavity so that each of the species occupies one half of the total area [Figure 4(a)]. In the second case we consider 15000 particles for species I and 5000 for species II, species I occupying three quarters of the total area and species II occupying one quarter of the cavity area [Figure 5(a)]. In the third and fourth cases [Figure 6(a) and 7(a)] we consider the same relative amounts of particles for the two species as in the second case, but locate them differently.

System 1 (Figure 4 ) is the only experiment where the two species are placed such that $\Omega_{1}, \Omega_{2}$, and $\Omega_{3}$ all lay on the interface. Thus, one can potentially achieve perfect mixing in the system at long mixing times. By contrast, in systems 2 and 3 illustrated in Figure 5 and 6, respectively, the interface does not cross any of the stagnation points and mixing is poor. System 4 (Figure 7) appears also to show good mixing properties, albeit not as good as system 1. In this case, the interface covers the stagnation point $\Omega_{1}$, but leaves the other stagnation points, $\Omega_{2}$ and $\Omega_{3}$, occupied only by one species.

\section{Mixing Quantification}

Once the description of the system had been obtained in terms of the motion of the fluids/particles, we performed the mixing analysis first by calculating the distributional entropy $S$ (location).

The influence of the scale of observation on mixing is explored by changing the number of bins. In Figure 8 we show the size and distribution of the bins for four different numbers of bins and the entropy $S$ (location) for the corresponding number of bins. The entropy has different values for different numbers of bins but when these values are normalized they all become equal. The normalized entropy is very close to 1 since $S$ (location) measures the overall distributive mixing irrespective of species and we consider well distributed particles from the starting configuration.

Since the distributional entropy $S$ (location) is constant at all scales of observation in the analysis, we can now study mixing by focusing on $S_{\text {location }}$ (species). In Figure 9(b), $S_{\text {location }}$ (species) is shown versus time for six numbers of bins: $100,400,900,1600,2500$, and 4900 . As a comparison the striation thickness behavior is also shown in Figure 9(a). To facilitate the comparison of the two plots, we exhibit on the $y$ axis ( 1 - striation thickness), the length 
a)

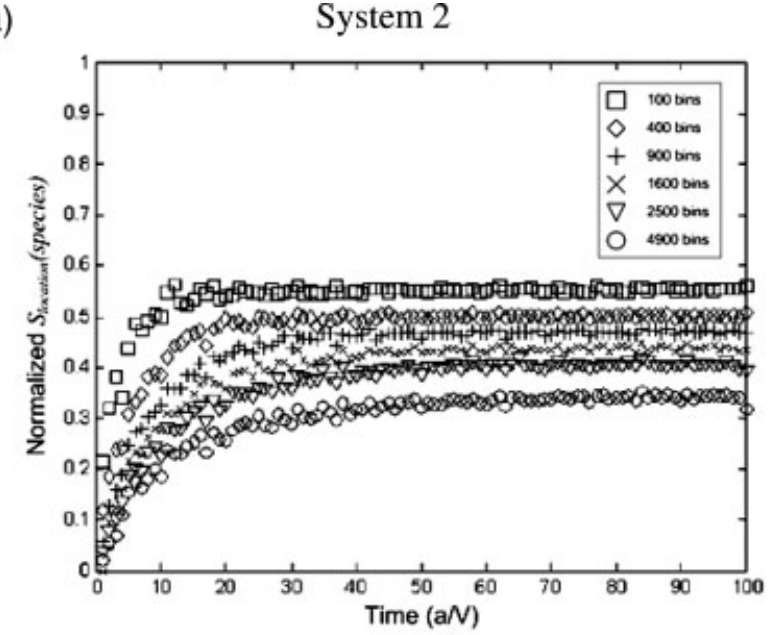

b)

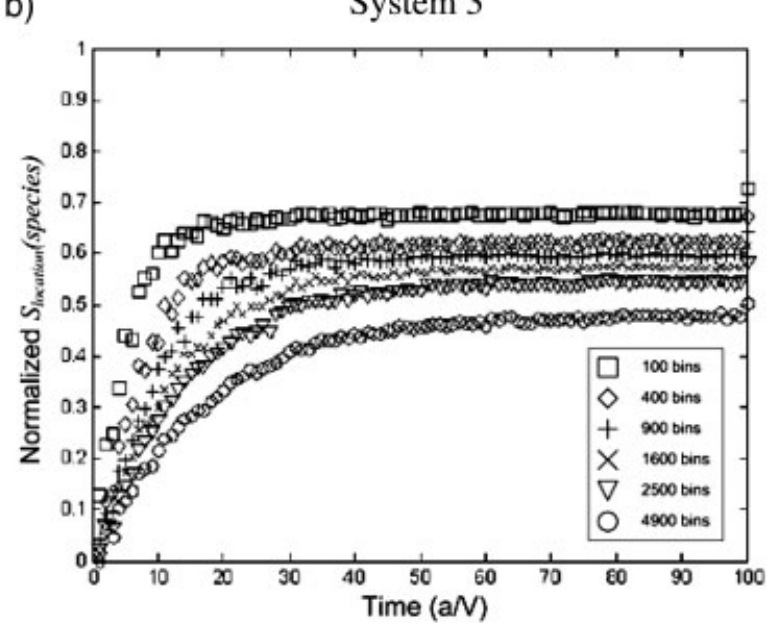

c)

System 4

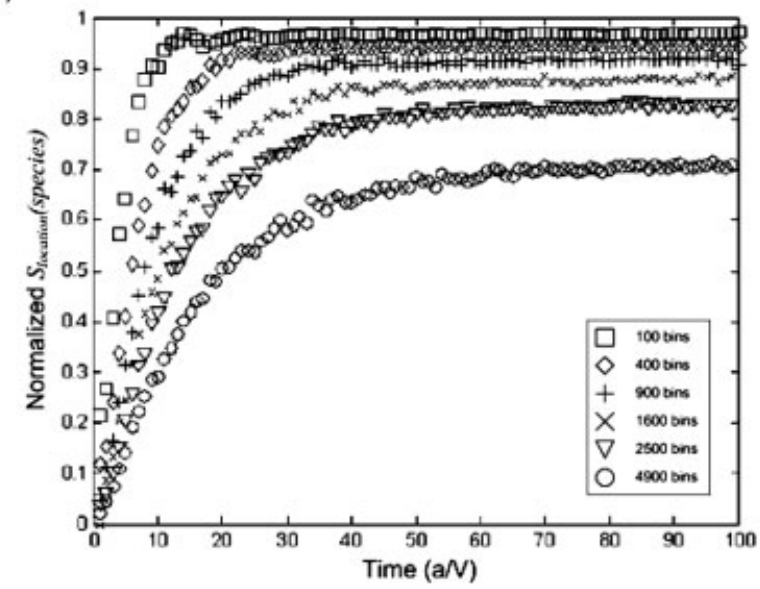

Figure 10. Dynamics of mixing for systems (a) system 2, (b) system 3, and (c) system 4; the difference between the systems clearly shows the effect of initial location on mixing.

being expressed in units of $a$. We note that the striationthickness method is prone to errors owing to uncertainty on the location of the interface as determined from a finite number of particles. In Figure 9 the measurements were taken by crossing with a horizontal line the left portion of the system at a height of $y=a / 2$ and measuring the width of the region occupied by one species closest to the left wall. For the systems 2, 3, and 4 the subjectivity of the striation thickness method becomes transparent since the result is greatly affected by the location of measurements.

The entropic method provides insight into the role of scale of observation in mixing analysis. As predicted, if the number of bins increases, the entropy decreases as the system is analyzed at smaller-length scales and the composition ratio of the two fluids in the bin can change. A second important effect of the number of bins is on the rate of mixing which is higher at a larger scale of observation (fewer bins). As shown in Figure 9(b), for 100 bins the species entropy achieves its maximum after about 20 time units, while, for 4900 bins, after 100 time units the species entropy is still growing and has not yet reached the plateau.

In Figure 10 we present the dynamics of mixing for systems 2,3 , and 4 in terms of the evolution of $S_{\text {location (species) with }}$ time using different scales of observation (different number of bins). The level of mixing achieved is poorer for systems 2 [Figure 10(a)] and 3 [Figure 10(b)] than for systems 1 [shown in Figure 9(b)] and 4 [Figure 10(c)].

A last analysis of the effect of the scale of observation on mixing is shown in Figure 11 in which we plot the normalized entropy $S_{\text {location }}$ (species) versus the number of bins for the four systems at different mixing times. In all cases the normalized $S_{\text {location (species) decreases as the number of }}$ bins increases. Although at shorter times the plots reach a plateau for a large enough number of bins, at longer times the plateau is not reached for our largest number of 4900 bins. 
a)

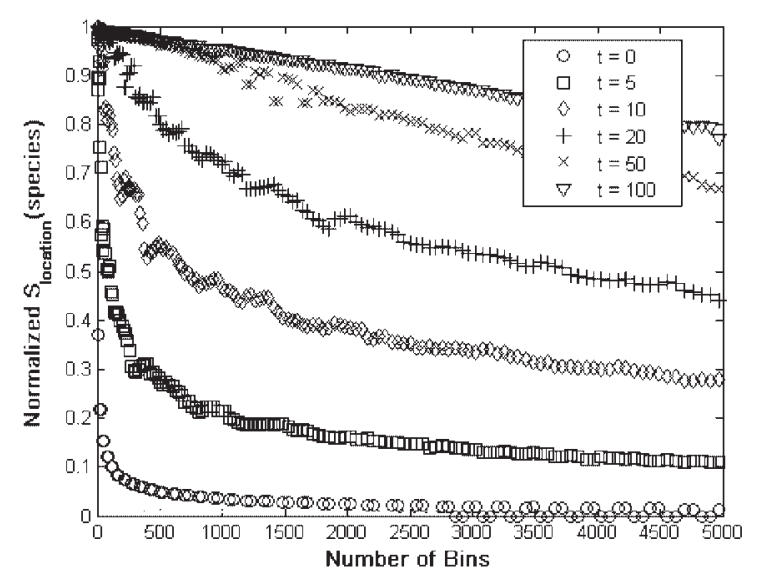

c)

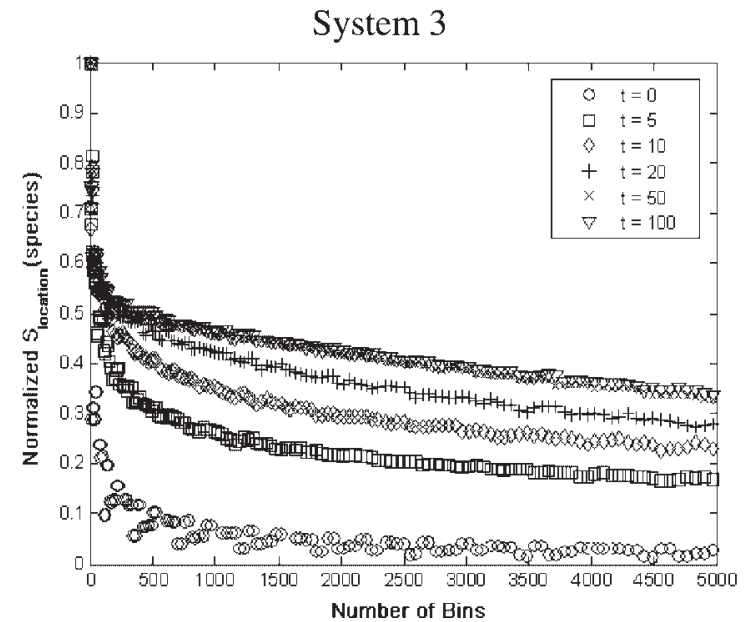

b)

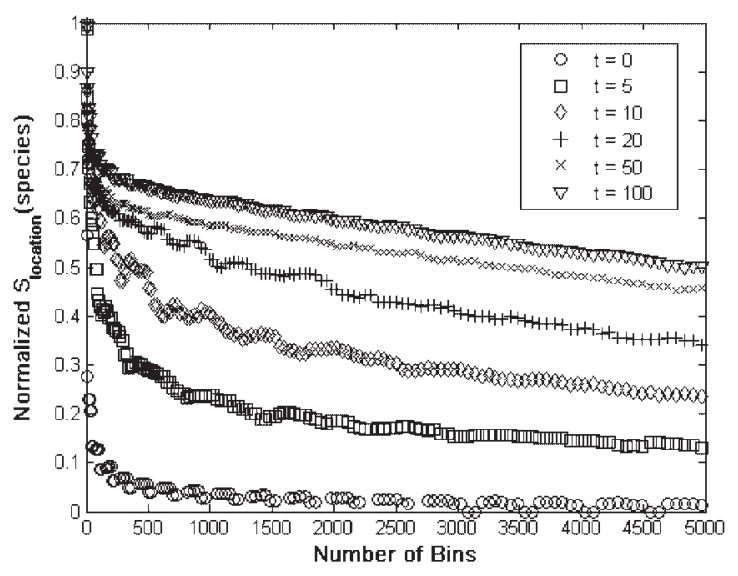

d)

System 4

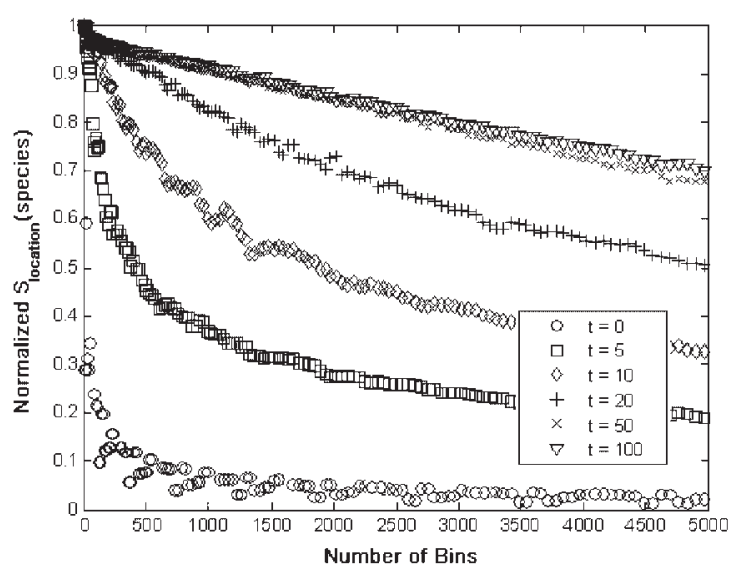

Figure 11. Normalized $S_{\text {location }}$ (species) versus the number of bins at different times (in units of $a / V$ ) for (a).system 1 , (b) system 2, (c) system 3, and (d) system 4 . The entropy always decreases as the number of bins increases but as the time increases the difference between the entropy calculated for a configuration with a low number of bins and the one calculated at a high number of bins decreases. As the time progresses the curves get closer indicating a change in the rate of mixing. For systems 3 and 4 there is no difference between the curves at $50 \mathrm{a} / \mathrm{V}$ and $100 \mathrm{a} / \mathrm{V}$, marking saturation in the level of mixing achieved at all scales of observation.

\section{Conclusion}

We have demonstrated that the use of statistical entropy offers a rigorous, practical, and efficient way of quantifying mixing for fluid systems.

By considering the Shannon entropy associated with the probability distribution of appropriately sized complexes/ groups of particles of various species, it is possible to quantify the mixing of several fluids. By plotting the conditional entropy $S_{\text {location }}$ (species) as a function of time we characterized and quantified the level of mixing of fluids in a system.

Finally, the effect of the scale of observation was studied by changing the number of bins used to calculate the entropy. By increasing this number it is possible to study smaller and smaller subdomains and a more exhaustive description of the system can be obtained. One cannot ignore the scale of observation when characterizing mixing.
The influence of the initial conditions on mixing was also considered. As our system exhibits nonchaotic, deterministic flow, the time evolution of the quality of mixing depends on the initial configuration.

Acknowledgements: The authors thank the National Science Foundation for the financial support of our research through grant DMI 0140412.

\section{Appendix}

The total entropy of the system is:

$$
S=-\sum_{j=1}^{M} \sum_{c=1}^{C}\left[p_{j, c} \ln p_{j, c}\right],
$$


where the joint probability $p_{j, c}$ is defined as:

$$
p_{j, c}=\frac{\frac{n_{j, c}}{P_{c}}}{\sum_{i=1}^{M} \sum_{c=1}^{C} \frac{n_{i, c}}{P_{c}}} .
$$

Because:

$$
p_{j, c}=p_{c / j} p_{j},
$$

where:

$$
p_{c / j}=\frac{\frac{n_{j, c}}{P_{c}}}{\sum_{c=1}^{C} \frac{n_{j, c}}{P_{c}}},
$$

and

$$
p_{j}=\frac{\sum_{c=1}^{C} \frac{n_{j, c}}{P_{c}}}{\sum_{i=1}^{M} \sum_{c=1}^{C} \frac{n_{i, c}}{P_{c}}} .
$$

Substituting Equation (A3) into Equation (A1):

$$
\begin{aligned}
S & =-\sum_{j=1}^{M} \sum_{c=1}^{C}\left[\left(p_{c / j} p_{j}\right) \ln \left(p_{c / j} p_{j}\right)\right] \\
& =-\sum_{j=1}^{M} \sum_{c=1}^{C}\left[\left(p_{c / j} p_{j}\right) \ln p_{j}\right]-\sum_{j=1}^{M} \sum_{c=1}^{C}\left[\left(p_{c / j} p_{j}\right) \ln p_{c / j}\right]
\end{aligned}
$$

But since $\sum_{c{ }_{1}}^{C} p_{c / j}=1$, Equation (6) in the main text can be written as:

$$
\begin{aligned}
S & =-\sum_{j=1}^{M}\left[p_{j} \sum_{c=1}^{C}\left(p_{c / j} \ln p_{c / j}\right)\right]-\sum_{j=1}^{M}\left[p_{j} \ln p_{j}\right] . \\
& \left.\left.=S_{\text {location }} \text { (species }\right)+S \text { (location }\right)
\end{aligned}
$$

The second form of Equation (A7) is reported as Equation (4) in the paper with the subsequent definition of the terms in Equation (5), (6), and (7).

[1] S. Wiggins, J. M. Ottino, Philos. Trans. R. Soc. London, A 2004, 362, 937.

[2] H. Aref, J. Fluid Mech. 1984, 143, 1.

[3] A. D. Stroock, S. K. W. Dertinger, A. Ajdari, I. Mezic, H. A. Stone, G. M. Whitesides, Science 2002, 295, 647.

[4] M. A. Burns, B. N. Johnson, S. N. Brahmasandra, K. Handique, J. R. Webster, M. Krishnan, T. S. Sammarco, P. M. Man, D. Jones, D. Heldsinger, C. H. Mastrangelo, D. T. Burke, Science 1998, 282, 484.
[5] S. W. Jones, W. R. Young, J. Fluid Mech. 1994, 280, 149.

[6] J. M. Ottino, S. Wiggins, Philos. Trans. R. Soc. London 2002 , 362, 923.

[7] P. V. Danckwerts, Appl. Sci. Res. A 1952, 3, 279.

[8] Z. B. Stone, H. A. Stone, Phys. Fluids 2005, 17, 063103.

[9] J. M. Ottino, "The Kinematics of Mixing: Stretching, Chaos and Transport", Cambridge University Press, Cambridge 1989.

[10] V. V. Meleshko, G. W. M. Peters, Phys. Lett. A 1996, 216, 87.

[11] R. Badii, A. Politi, "Complexity. Hierarchical Structures and Scaling in Physics", Cambridge University Press, Cambridge 1997.

[12] D. Therriault, S. R. White, J. A. Lewis, Nat. Mater. 2003, 2 , 265.

[13] F. J. Muzzio, P. D. Swanson, J. M. Ottino, Phys. Fluids A 1991, 3, 822 .

[14] M. Liu, R. L. Peshkin, F. J. Muzzio, C. W. Leong, AIChE J. 1994, 40, 1273.

[15] C. L. Tucker, G. W. M. Peters, Korea Australia Rheology J. 2003, 15, 197.

[16] W. J. A. Dahm, P. E. Dimotakis, AIAA J. 1987, 25, 1216.

[17] F. Bottausci, I. Mezic, C. Meinhart, C. Cardonne, Philos. Trans. R. Soc. London 2004, 362, 1001.

[18] L. K. Su, N. T. Clemens, Exp. Fluids 1999, 27, 507.

[19] B. R. Noack, I. Mezic, G. Tadmor, A. Banaszuk, Physics of Fluids 2004, 16, 867.

[20] C. E. Shannon, Bell Syst. Tech. J. 1948, 27, 379.

[21] A. I. Khinchin, "Mathematical Foundations of Information Theory", Dover, New York 1957.

[22] P. A. Allen, M. D. Murphy, M. Kaufman, K. E. Groth, A. Begovic, J. Geront. B: Psych. Sci. Soc. Sci. 2004, 59, 210.

[23] D. R. Brooks, "Evolution as Entropy", University of Chicago Press, Chicago 1998.

[24] R. Everson, D. Manin, L. Sirovich, M. Winter, AIAA J. 1998, 36,121 .

[25] N. Georgescu Roegen, "The Entropy Law and the Economic Process", Harvard University Press, Cambridge 1971.

[26] P. Grassberger, I. Procaccia, Physica D 1984, 13, 34.

[27] W. Wang, I. Manas Zloczower, M. Kaufman, AIChE J. 2003, 49,1644

[28] W. Wang, I. Manas Zloczower, M. Kaufman, Int. Polym. Proc. 2001, 16, 1.

[29] K. Alemaskin, M. Camesasca, I. Manas Zloczower, M. Kaufman, E. K. Kim, M. Spalding, W. Trumbull, R. Swain, ANTEC Conference Proceedings 2004.

[30] M. Camesasca, I. Manas Zloczower, M. Kaufman, Plast. Rubber Compos. 2004, 33, 372.

[31] M. Camesasca, I. Manas Zloczower, M. Kaufman, J. Micro mech. Microeng. 2005, 15, 2038.

[32] Z. Tadmor, C. G. Gogos, "Principles of Polymer Proces sing", John Wiley, New York 1979.

[33] M. Camesasca, Ph.D. Thesis, Case Western Reserve University, 2006.

[34] P. D. Swanson, J. Ottino, J. Fluid Mech. 1990, 213, 227.

[35] W. Linxiang, F. Yurun, Int. J. Bifurcation Chaos 2001, 11, 1953. 